\title{
EDITORIAL
}

Yukio Miyagawa $\cdot$ Makoto Tsuchimochi

\section{Odontology receives an impact factor}

We are very pleased to announce that Odontology has received its first impact factor, 1.833 , according to Thomson Reuters Journal Citation Reports (JCR) 2008. The impact factor for Odontology ranked 21st among 55 JCR journals in the subject categories of dentistry, oral surgery, and medicine. In this way, Odontology is being recognized for the high quality of the peer-reviewed articles published in the journal. We would like to thank authors, reviewers, members of the editorial board, and the publisher for their support and dedication in maintaining a high standard for the journal. We are also grateful to The Nippon Dental University for its generous support of our biannual publication, which was inaugurated in 2009. Odontology will continue to be at the forefront in disseminating novel, highquality findings of advanced work from many researchers and clinicians in dental science and related fields. We look forward to receiving your submissions and your comments.
Y. Miyagawa

President, The Society of The Nippon Dental University

Division of Biomaterials, Advanced Research Center, The Nippon

Dental University School of Life Dentistry at Niigata, Niigata, Japan

M. Tsuchimochi $(\bowtie)$

Editor-in-Chief

Department of Oral and Maxillofacial Radiology, The Nippon

Dental University School of Life Dentistry at Niigata,

1-8 Hamaura-cho, Chuo-ku, Niigata, Niigata 951-8580, Japan

Tel. +81-25-267-1500; Fax +81-25-233-0307

e-mail: tsuchimochi@ngt.ndu.ac.jp 